\title{
Neuralgic amyotrophy: A rare cause of bilateral diaphragmatic paralysis
}

\author{
Neil Shinder MD FRCPC, Alasdair Polson MD FRCPC, Elizabeth Pringle MD FRCPC, \\ Denis E O'Donnell MD FRCPI FRCPC \\ Division of Respiratory and Critical Care Medicine, Queen's University, Kingston and Division \\ of Neurology, University of Ottawa, Ottawa, Ontario
}

\begin{abstract}
N Shinder, A Polson, E Pringle, DE O’Donnell. Neuralgic amyotrophy: A rare cause of bilateral diaphragmatic paralysis. Can Respir J 1998;5(2):139-142.

Neuralgic amyotrophy, also known as brachial neuritis, is a well described clinical entity. Diaphragmatic dysfunction, as a result of phrenic nerve root involvement (cervical roots 3 to 5), is an uncommon, but increasingly recognized association. The case of a previously healthy 61-year-old woman who, after a prodrome of neck and shoulder discomfort, presented with severe orthopnea is described. Pulmonary function and electrophysiological studies led to a diagnosis of bilateral diaphragmatic paralysis. The patient's clinical course and the exclusion of other nerve entrapment syndromes and neurological disorders strongly favoured the diagnosis of neuralgic amyotrophy.
\end{abstract}

Key Words: Bilateral diaphragmatic paralysis, Brachial neuritis, Dyspnea, Neuralgic amyotrophy, Orthopnea

\section{La névralgie amyotrophique : une rare cause de paralysie bilatérale du diaphragme}

RÉSUMÉ : La névralgie amyotrophique ou névrite brachiale est une entité clinique bien documentée. La dysfonction diaphragmatique, résultant d'une atteinte de la racine du nerf phrénique (racines cervicales 3 à 5), est rarement associée à ce syndrome mais on la reconnaît de plus en plus souvent. On décrit le cas d'une femme de 61 ans, auparavant en bonne santé qui, après un prodrome de douleur au cou et à l'épaule, a présenté une orthopnée sévère. Les études électrophysiologiques et de la fonction pulmonaire ont conduit au diagnostic de paralysie diaphragmatique bilatérale. L'évolution clinique de la patiente et l'exclusion des autres syndromes de compression des nerfs et de troubles neurologiques ont fortement appuyé le diagnostic de névralgie amyotrophique.
$\mathrm{W}$ describe a 61-year-old woman who presented with sudden onset of acute severe dyspnea two weeks after undergoing an elective surgical procedure. Dyspnea, in subsequent weeks, progressed to a level where it became inca- pacitating. Acute bilateral diaphragmatic paralysis secondary to neuralgic amyotrophy (NA) was eventually diagnosed. This report summarizes the differential diagnosis, clinical course and management of this rare condition.

Presented at the Ontario Thoracic Society's Annual Meeting, Toronto, Ontario, February 2, 1996; awarded “Best Clinical Presentation" by a Respiratory Trainee in Ontario

Correspondence: Dr DE O'Donnell, Richardson House, 102 Stuart Street, Kingston, Ontario K7L 2V6. Telephone 613-545-6328, fax 613-549-1459, e-mail odonnell@ post.queensu.ca 


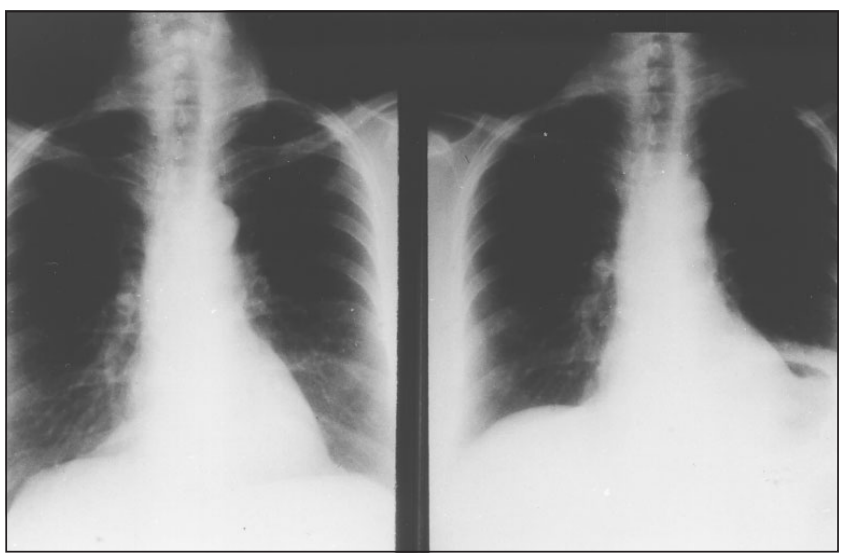

Figure 1) Preoperative chest radiograph (left) and postoperative films one month later (right). Note elevated hemidiaphragms and left basal atelectasis

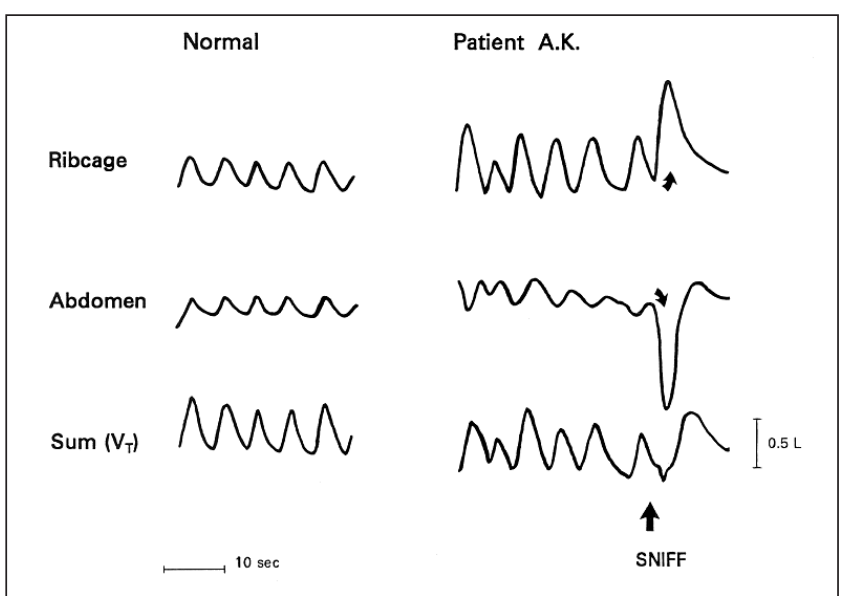

Figure 2) Respitrace volume tracing during spontaneous breathing and a maximal sniff manoeuvre in patient (right) and in a normal subject (left). Note paradoxical abdominal breathing. $V_{T}$ Tidal volume

\section{CASE PRESENTATION}

Four days after an elective, uncomplicated hysterectomy for a benign condition, a 61-year-old woman complained of sudden onset of neck pain radiating to her right shoulder. The patient was thought to have arthritis of the cervical spine, and analgesics were prescribed with consequent symptomatic benefit. However, after discharge from hospital, her neck discomfort persisted and worsened, causing the patient to seek assistance from a registered physiotherapist who administered gentle manual traction of the cervical spine as well as neck retraction exercises. The neck discomfort improved, but one week into therapy and $24 \mathrm{~h}$ after her last physiotherapy session, the patient developed sudden severe dyspnea that limited her daily activities. She was readmitted to hospital where a chest $\mathrm{x}$-ray showed elevated hemidiaphragms and bibasal atelectasis, which was predominantly left-sided; her preoperative chest x-ray was entirely normal (Figure 1). Arterial blood gases showed $\mathrm{PaO}_{2}$ of $69.0 \mathrm{mmHg}, \mathrm{PaCO}_{2}$ $33.0 \mathrm{mmHg}$ and $\mathrm{pH}$ 7.44. A ventilation-perfusion scan showed low probability for pulmonary embolism, and bilateral leg angiodynography was normal. However, because of

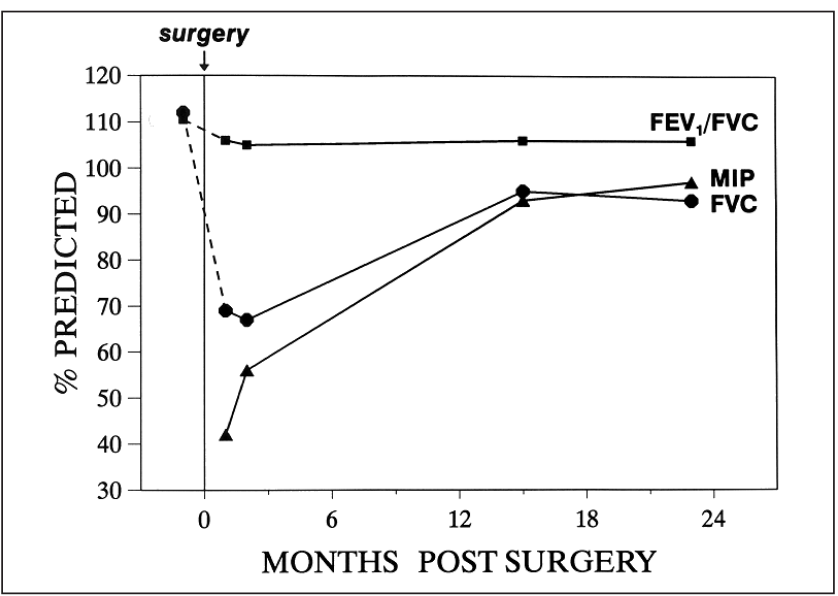

Figure 3) Trend graphs of spirometric indexes and maximal inspiratory pressure over a 23-month follow-up. FEV ${ }_{1}$ Forced expiratory volume in $1 \mathrm{~s}$ (predicted values are from references 11 and 12); FVC Forced vital capacity; MIP Maximal inspiratory pressure

a strong clinical suspicion of pulmonary embolism, the patient was temporarily placed on anticoagulant therapy, and arrangements were made for follow-up with a respirologist.

On respiratory consultation, it was determined that the patient now had severe orthopnea and was unable to lie flat. This resulted in poor sleep quality and subjective exhaustion. She also reported a four-week history of exertional dyspnea with curtailment of exercise capacity. She reported anterior and lateral chest wall discomfort, as well as upper abdominal discomfort. Her neck pain had improved considerably over a two-week interval. There was no cough, sputum production, hemoptysis, swallowing difficulty or symptoms to suggest lower respiratory tract infection. Past medical history was unremarkable apart from a diagnosis of a postviral cough six months previously, which had resolved.

Examination revealed increased accessory muscle activity, diminished lower thoracic respiratory excursions and evidence of thoracoabdominal paradoxical breathing in the supine position (Figure 2) - a position that she could only tolerate for approximately $1 \mathrm{~min}$. She also had diminished breath sounds at the left base on auscultation and an impaired percussion note at this site. Neurological examination was normal. There was no sensory deficit and no observed fasciculation or muscle wasting. There was no muscle weakness apart from appreciable weakness of the left rhomboid muscle. Examination of the cardiovascular system was normal.

Pulmonary investigations: Pulmonary function tests (PFTs) showed a restrictive ventilatory deficit compared with baseline PFTs six months earlier (total lung capacity $[\mathrm{TLC}]=5.73 \mathrm{~L}[117 \%$ predicted $]$, forced vital capacity $[\mathrm{FVC}]=3.42 \mathrm{~L}[112 \%$ predicted $]$, forced expiratory volume in $1 \mathrm{~s}=2.81 \mathrm{~L}$ [ $126 \%$ predicted]) when she was diagnosed with postviral cough. TLC and FVC decreased by $31 \%$ and $39 \%$, respectively, from the baseline study (Figure 3). Diffusion capacity was normal $(21.7 \mathrm{~mL} / \mathrm{min} / \mathrm{mmHg}, 98 \%$ predicted). Maximal inspiratory pressure was $30 \mathrm{~cm} \mathrm{H}_{2} \mathrm{O}$ measured at functional residual capacity ( $42 \%$ predicted 
maximum). Maximal expiratory pressures were normal. FVC and $\mathrm{PaO}_{2}$ decreased by $0.99 \mathrm{~L}$ (ie, $51 \%$ ) and $14 \mathrm{mmHg}$, respectively on assuming the supine position from a sitting position. Diaphragmatic fluoroscopy showed paradoxical upward movement of both hemidiaphragms on sniffing, confirming the presence of bilateral diaphragmatic paralysis. Nocturnal polysomnography showed normal sleep architecture with some REM-related hypopneic events (less than $2 \mathrm{~h}$ ) associated with unsustained arterial oxygen desaturation to $81 \%$. The entire sleep study was conducted with the patient lying in her preferred left lateral position. She could not tolerate the supine position.

Neurological investigations: Cervical spine radiograph showed some degenerative changes and osteophyte formation at the $\mathrm{C} 2$ to $\mathrm{C} 7$ level, with minor $\mathrm{C} 6$ to $\mathrm{C} 7$ disc space narrowing. Computed togmography (CT) myelogram did not reveal cervical root impingement or spinal cord compression. CT of the thorax was normal. Nuclear magnetic resonance imaging of the cervical spine and brachial plexus was contraindicated because of a previous metal stapedial implant. Needle electromyography (EMG) of the right and left hemidiaphragms showed fibrillation potentials and positive sharp waves. No voluntary motor units were seen in the left hemidiaphragm. In the right hemidiaphragm, normal appearing motor units were seen, although they were reduced in number and firing rapidly with each respiration (ie, neurogenic pattern of recruitment). There was also denervation, as shown by fibrillation potentials and positive sharp waves in the cervical paraspinal muscles, on the left at the $\mathrm{C} 4, \mathrm{C} 5$ and C6 levels, most marked at the C4 level. Needle EMG of the left rhomboids also showed abnormal spontaneous activity in the form of fibrillation potentials and positive sharp waves, as well as neurogenic recruitment of normal appearing motor units. Needle EMG of the left biceps was normal.

Clinical course: The patient was started on nocturnal oxygen therapy and later on bilevel positive airway pressure (BIPAP) (Respironics Inc, Pennsylvania) (inspiratory pressure $8 \mathrm{~cm} \mathrm{H} \mathrm{H}_{2} \mathrm{O}$; expiratory pressure $3 \mathrm{~cm} \mathrm{H}_{2} 0$ ) during sleep. This allowed the patient to sleep comfortably in the supine position, with consequent subjective improvement in sleep quality and daytime fatigue and amelioration of dyspnea. Symptoms improved over the ensuing 15 months, with concomitant improvements in the chest x-ray findings, static lung volumes and inspiratory muscle strength measurements (Figure 3). BIPAP and oxygen were discontinued after 3.5 months. Repeat electrophysiological studies four months after the original study showed marked improvement (ie, bilateral phrenic nerve stimulation studies showed normal responses with motor terminal latencies now in the normal range). Needle EMG of hemidiaphragms, cervical paraspinal muscles and left rhomboids showed multiple large amplitude polyphasic motor unit potentials. No fibrillation potentials or positive sharp waves were present. These studies confirmed the presence of widespread reinnervation of the diaphragm and cervical paraspinal muscles, with no evidence of remaining active denervation. However, at 15 months, diaphragm function remained impaired as demonstrated by re- duced maximal transdiaphragmatic pressures (Pdimax occlusion $36 \mathrm{~cm} \mathrm{H}_{2} \mathrm{O}$ ) (normal range for women 82 to 182 $\mathrm{cm} \mathrm{H}_{2} \mathrm{O}$ ).

\section{DISCUSSION}

The sudden onset of severe dyspnea, hypoxemia and new radiographic abnormalities in a patient recovering from surgery gave rise to clinical suspicion of pulmonary embolism. Subsequent evaluation, however, determined that the predominant symptom was orthopnea, and prompted a number of pulmonary and neurophysiological investigations that confirmed a diagnosis of bilateral diaphragmatic paralysis. The differential diagnosis for this clinical presentation is large, but the main considerations were nerve compression injury, neuromuscular disease (eg, motor neuron disease) and NA.

The patient had evidence of degenerative cervical osteoarthritis with osteophyte formation, which may have predisposed her to a nerve traction injury due to neck hyperextension during surgical intubation or due to subsequent cervical manipulation during physiotherapy. There is one previously published case of unilateral diaphragmatic paralysis following chiropractic manipulation of the cervical spine (1). In that instance, neck pain and dyspnea followed immediately after vigorous head rotation and neck flexion exercises (1). Our patient's intubation during surgery was uneventful, and neck and shoulder pain first occurred four days after surgery with the acute onset of dyspnea occurring approximately two weeks later. The patient's persistent neck and shoulder discomfort precluded any manipulation other than gentle manual traction of the cervical spine by the physiotherapist. The abrupt onset of breathlessness did not occur at the time of physiotherapy, but happened one day after the last physiotherapy session. Finally, the CT myelogram ruled out cervical spondylosis or disc protrusion: there was no evidence of compression of the spinal cord or cervical nerve roots. CT of the thorax did not reveal any site of compression of the phrenic nerves along their anatomic courses.

Neurological examination, the results of electrophysiological studies and the subsequent clinical course ruled out the possibility of motor neuron disease or other neuromuscular disease (inflammatory or vasculitic) as an explanation for brachial nerve paresis. The onset of orthopnea due to bilateral diaphragmatic paralysis after a prodrome of acute neck and shoulder pain, together with the electromyelographic evidence of axonal degeneration of the brachial plexus, are features that strongly suggeste a diagnosis of NA (2-4).

The term 'neuralgic amyotropy' was first used in 1948 by Parsonage and Turner (5) to describe brachial neuritis of unknown origin, characterized by neck and shoulder pain with the later development of motor weakness over a variable period of one to 28 days. Subsequently, associated radiographic evidence of unilateral or bilateral diaphragmatic paralysis became a documented association occurring in up to $7 \%$ of patients in one series (6). Recently, the results of more extensive electrophysiological evaluation of diaphragmatic dysfunction in this condition have been published (3).

The etiology of NA remains unknown, but immunological 
mechanisms have been implicated (7-9). A proportion of cases were associated with prior respiratory tract infections, vaccines, toxoids, interferon therapy and, interestingly, prior surgery (7-9). The condition is more common in men (male:female ratio of $2.4: 1$ ), with only a few cases previously reported in females (6). Although the outlook for NA without phrenic nerve involvement is good, the prognosis for a complete recovery of diaphragmatic function in those with phrenic involvement is uncertain, and relapses have been documented (10). Mulvey et al (3) recently reported the results of follow-up in five patients with bilateral phrenic paralysis due to NA, none of whom have shown complete recovery during a follow-up period of two to four years after initial presentation.

The use of noninvasive ventilation in the management of this condition has not previously been reported. While supplemental oxygen therapy abolished nocturnal arterial oxygen desaturations, the present patient did not derive symptomatic benefit from this alone. The addition of nocturnal rest therapy with BIPAP, however, proved to be a useful intervention that improved sleep quality, reduced general fatigue, relieved exertional dyspnea and improved exercise tol-

\section{REFERENCES}

1. Heffner E. Diaphragmatic paralysis following chiropractic manipulation of the cervical spine. Arch Intern Med 1985;145:562-4.

2. Walsh N, Dumutr D, Kalantri A, Roman A Jr. Brachial neuritis involving the bilateral phrenic nerves. Arch Phys Med Rehab 1987;68:46-8.

3. Mulvey D, Aquilina R, Elliott M, Moxham J, Green M. Diaphragmatic dysfunction in neuralgic amyotrophy: An electrophysiologic evaluation of 16 patients presenting with dyspnea. Am Rev Respir Dis 1993;147:66-71.

4. Pandit A, Kalra S, Woodcock A. An unusual cause of bilateral diaphragmatic paralysis. Thorax 1992;47:201.

5. Parsonage MJ, Turner JWA. Neuralgic amyotrophy: the shoulder-girdle syndrome. Lancet 1948;i:973-8.

6. Tsairis P, Dyck P, Mulder D. Natural history of brachial plexus neuropathy: report on 99 patients. Arch Neurol 1972;27:109-17.

7. Reutens DC, Dunne JW, Leather H. Neuralgic amyotrophy following erance. Moreover, BIPAP therapy relieved the chest wall and abdominal discomfort, presumably by resting overburdened intercostal, accessory and abdominal muscles. Diaphragmatic function improved significantly over the 23 months of follow-up in this patient, as shown by improved symptoms, vital capacity measurements, phrenic nerve conduction studies and static inspiratory muscle strength measurements. However, there was evidence of residual diaphragmatic dysfunction (reduced transdiaphragmatic pressures) 15 months after presentation and vital capacity had not reached baseline values at 23 months. There is insufficient information in the literature to predict this patient's prospect of a complete recovery with time.

In summary, we believe that NA is the most likely cause for this patient's diaphragmatic dysfunction. The pattern of clinical presentation and the results of the electrophysiological studies fit well with the previous descriptions of this clinical syndrome, particularly in the absence of evidence of neuromuscular disease or cervical root entrapment. NA should be considered in the differential diagnosis in any previously healthy patient who presents with neck discomfort and subsequent sudden orthopnea.

recombinant DNA hepatitis B vaccination. Muscle Nerve 1990;13:461. (Lett)

8. Bernsen PL, Wong-Chung RE, Vingerhoets HM, Janssen JT. Bilateral neuralgic amyotrophy induced by interferon therapy. Arch Neurol 1988;45:449-51.

9. Sierra A, Prat J, Bas J, et al. Blood lymphocytes are sensitised to brachial plexus nerves in patients with neuralgic amyotrophy. Acta Neurol Scand 1991;83:183-6.

10. Graham AN, Martin PD, Haas LF. Neuralgic amyotrophy with bilateral diaphragmatic palsy. Thorax 1985;40:635-6.

11. Goldman HI, Becklake MR. Respiratory function tests: normal values at median altitudes and the prediction of normal results. Am Rev Tuberc Pulm Dis 1959;79:457-67.

12. Black LF, Hyatt RE. Maximal respiratory pressure: Normal values and relationship to age and sex. Am Rev Respir Dis 1969;99:696-702. 


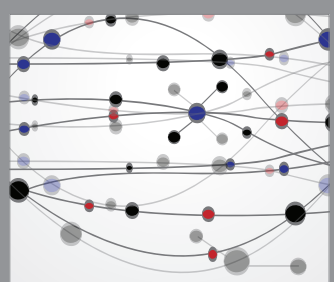

The Scientific World Journal
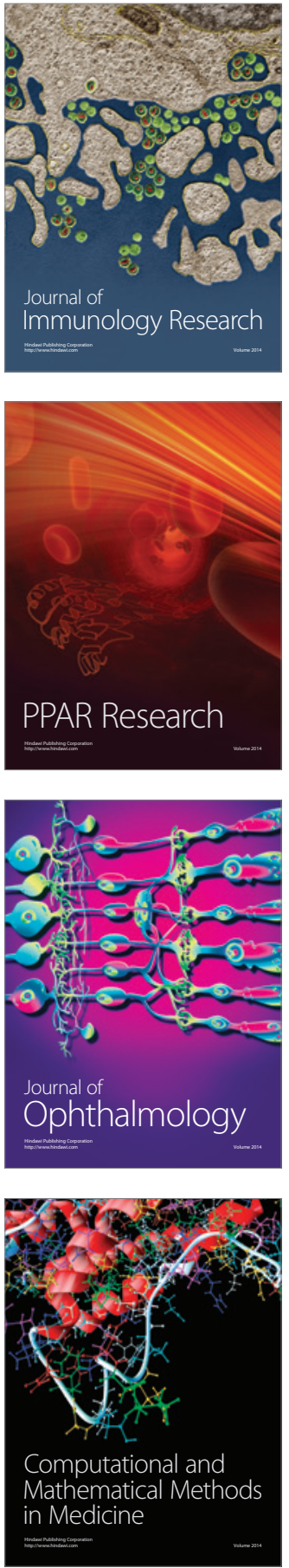

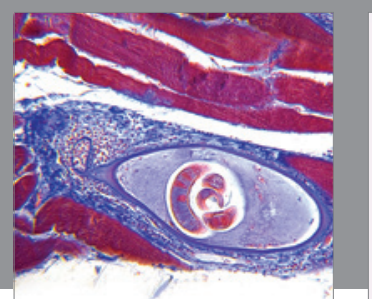

Gastroenterology Research and Practice

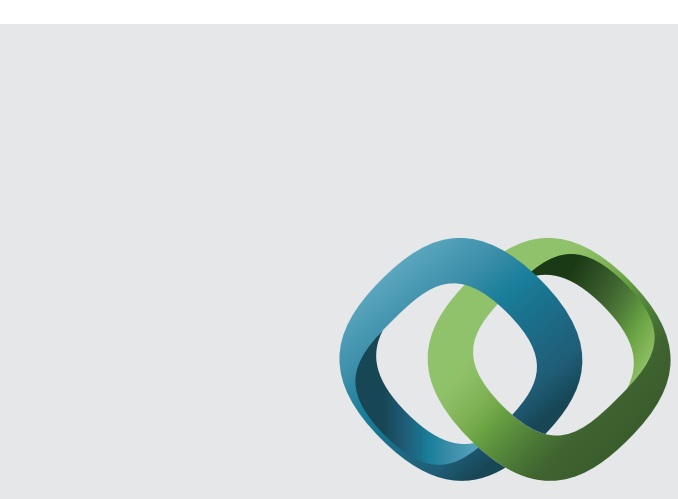

\section{Hindawi}

Submit your manuscripts at

http://www.hindawi.com
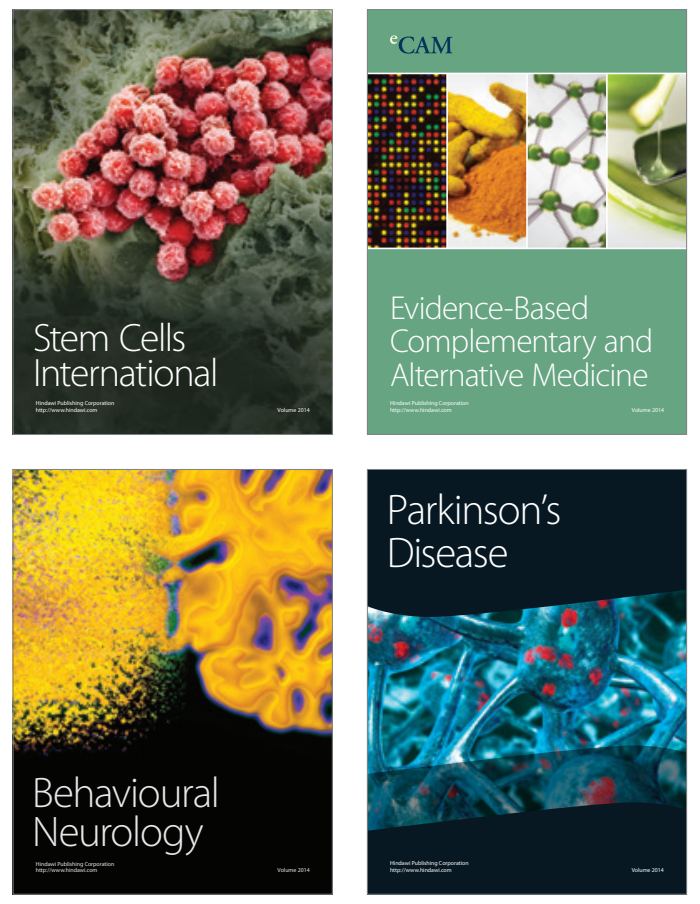
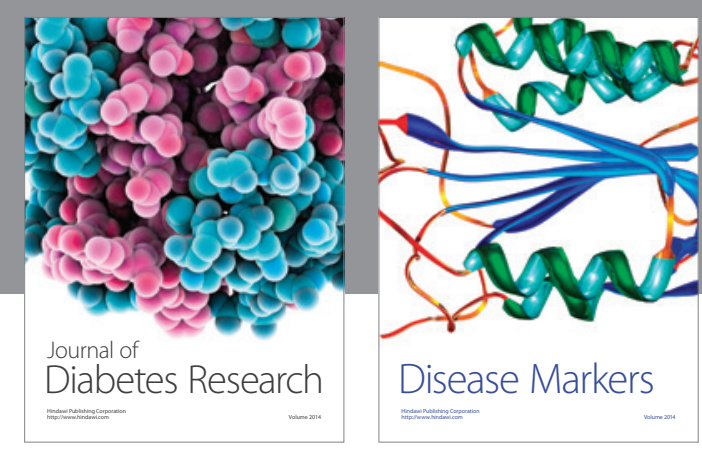

Disease Markers
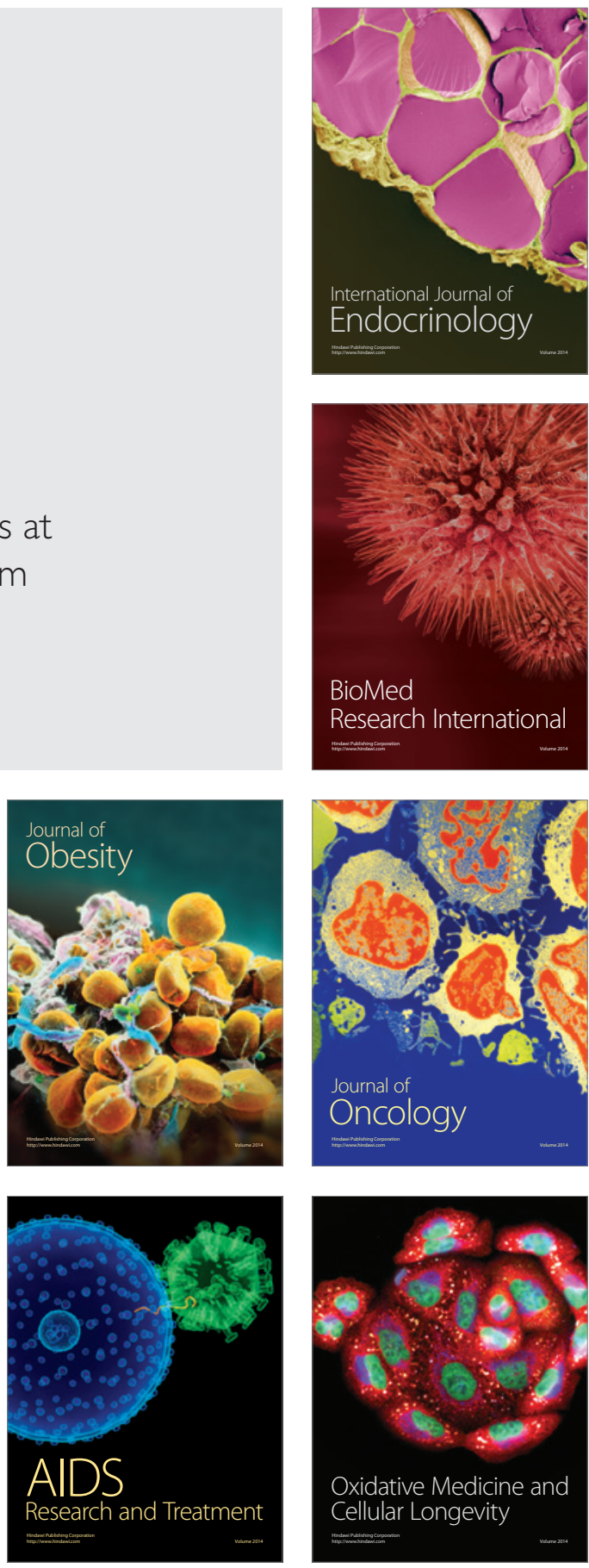